\title{
Classical Electromagnetic Field Theory in the Presence of Magnetic Sources
}

\author{
Wen-Jun Chen, ${ }^{a}$ Kang Lia ${ }^{a}$ and Carlos M. Naón $\left.{ }^{b}\right]$ \\ ${ }^{a}$ Zhejiang Institute of Modern Physics, Zhejiang University, Hangzhou, 310027, P.R. China \\ ${ }^{b}$ Instituto de Física La Plata, Departamento de Física, Facultad de Ciencias Exactas, \\ Universidad Nacional de La Plata, CC 67,1900 La Plata, Argentina
}

\begin{abstract}
Using two new well defined 4-dimensional potential vectors, we formulate the classical Maxwell's field theory in a form which has manifest Lorentz covariance and $S O(2)$ duality symmetry in the presence of magnetic sources. We set up a consistent Lagrangian for the theory. Then from the action principle we get both Maxwell's equation and the equation of motion of a dyon moving in the electromagnetic field.
\end{abstract}

PACS: 03.50.De, 11.30.-j, 14.80.Hv

\footnotetext{
${ }^{1}$ kangli@zimp.zju.edu.cn

${ }^{2}$ naon@venus.fisica.unlp.edu.ar
} 


\section{Introduction}

Recently there has been an increasing interest in the study of electromagnetic (EM) duality symmetry, because it plays a fundamental role in superstring and brane theory [1] [2]. From Maxwell's equations we know that general EM duality implies the existence of magnetic source ( magnetic charge (monopole) and currents). However, when considering the quantum dynamics of particles carrying both electric and magnetic charges (dyons) one faces the lack of a naturally defined classical field theory despite of the fact that a consistent quantum field theory does exist [3]. This issue was analyzed in recent contributions of many authors [5] [6] [6] 8] [9]. In our recent paper [14], we gave an alternative formulation of electric-magnetic field theory in the presence of magnetic source. The advantages of our formulation are the following. First, we introduce two new potential vectors that have no singularities and we do not need to use the concept of Dirac String; secondly, from the present paper we can set up a consistent Lagrangian theory from which we can get all the information about classical electromagnetic field theory which returns to the usual Maxwell's field theory when only electric source is considered; thirdly it has manifest Lorentz covariant and $S O(2)$ duality symmetry. Finally it seems that our formulation can be quantized directly, an issue that will be reported in a forthcoming article.

The aim of this paper is to present the details of the construction of a Lagrangian for the EM field theory in the formulation of [14]. From the action principle we expect to get the Maxwell's equation and also the equation of motion of a dyon moving in the electro-magnetic field. We also explain also why our formalism has manifestly $S O(2)$ duality symmetry. The paper is organized as follows. In the next section we give a brief review of our formulation of classical electromagnetic field in the presence of magnetic source, where two well defined 4-dimensional potential vectors are introduced and the Maxwell's equations are written in a Lorentz covariant way. In the third section we show how manifest $S O(2)$ duality symmetry arises in the present approach. In section 4, we

give the Lagrangian form of our formulation. In section 5, from the action principle of the system of a dyon, we get both Maxwell's equation and the equation of motion of the dyon. Some conclusion remarks are given in the last section. 


\section{Two potential vectors formulation}

Let us first give a brief review of the two 4-vector potentials formulation of the electromagnetic field in the presence of magnetic source 14. Besides the usual definition of 4-dimensional potential which we called $A_{\mu}^{1}$, i.e.

$$
A_{\mu}^{1}=\left(\phi_{1},-\mathbf{A}_{1}\right), \text { or } A^{\mu 1}=\left(\phi_{1}, \mathbf{A}_{\mathbf{1}}\right)
$$

we also introduce

$$
A_{\mu}^{2}=\left(\phi_{2},-\mathbf{\mathbf { A } _ { 2 }}\right), \text { or } A^{\mu 2}=\left(\phi_{2}, \mathbf{\mathbf { A } _ { 2 }}\right),
$$

where $\phi_{1}$ and $\mathbf{A}_{1}$ are usual electric scalar potential and magnetic vector potential in electrodynamics, while the newly introduced potential $\phi_{2}$ is the scalar potential associated to the magnetic field and $\mathbf{A}_{2}$ is a vector potential associated to the electric field. It should be stressed that these two 4-potentials have no singularities around the magnetic charges (monopoles). Using these potentials, the electric field strength $\mathbf{E}$ and the magnetic induction $\mathbf{B}$ are then expressed as:

$$
\begin{gathered}
\mathbf{E}=-\nabla \phi_{1}-\frac{\partial \mathbf{A}_{\mathbf{1}}}{\partial t}+\nabla \times \mathbf{A}_{\mathbf{2}}, \\
\mathbf{B}=\nabla \phi_{2}+\frac{\partial \mathbf{A}_{\mathbf{2}}}{\partial t}+\nabla \times \mathbf{A}_{\mathbf{1}} .
\end{gathered}
$$

In the magnetic source free case, $\phi_{2}$ and $\mathbf{A}_{2}$ are expected to be zero, so the above equation returns to the usual magnetic source free case.

Now we introduce two field tensors as

$$
F_{\mu \nu}^{I}=\partial_{\mu} A_{\nu}^{I}-\partial_{\nu} A_{\mu}^{I}, \quad I=1,2 .
$$

Then, choosing Lorentz gauge $\partial^{\mu} A_{\mu}^{I}=0$, the Maxwell's equation in the case of existing both electric and magnetic sources,

$$
\begin{array}{cl}
\nabla \cdot \mathbf{E}=\rho_{e}, & \nabla \times \mathbf{B}=\mathbf{j}_{\mathbf{e}}+\frac{\partial \mathbf{E}}{\partial t}, \\
\nabla \cdot \mathbf{B}=\rho_{m}, & \nabla \times \mathbf{E}=-\mathbf{j}_{\mathbf{m}}-\frac{\partial \mathbf{B}}{\partial t},
\end{array}
$$

can be recast as

$$
\partial^{\mu} F_{\mu \nu}^{I}=g^{I I^{\prime}} J_{\nu}^{I^{\prime}}
$$

where

$$
g^{I I^{\prime}}=\left(\begin{array}{cc}
1 & 0 \\
0 & -1
\end{array}\right) \text {. }
$$


and

$$
J_{\mu}^{1}=J_{\mu}^{e}=\left(\rho_{e},-\mathbf{j}_{\mathbf{e}}\right), \quad J_{\mu}^{2}=J_{\mu}^{m}=\left(\rho_{m},-\mathbf{j}_{\mathbf{m}}\right) .
$$

In this formulation the currents are manifestly conserved:

$$
\partial^{\nu} J_{\nu}^{I} \propto \partial^{\nu} \partial^{\mu} F_{\mu \nu}^{I}=0
$$

In the next section, we will see that the index $I$ is the $S O(2)$ index, so our formulation above has manifestly $S O(2)$ duality symmetry which is related to the general gauge transformation $A_{\mu}^{I} \rightarrow A_{\mu}^{I}+\partial_{\mu} \chi^{I}$. The fields $\mathbf{E}, \mathbf{B}$, the field tensors in (2.5) and Maxwell's equations (2.8) are all invariant under the transformations.

Let us stress also that in the expressions above neither $F_{\mu \nu}^{1}$ nor $F_{\mu \nu}^{2}$ have the same matrix form as the usual electromagnetic tensor. From (2.5) and the definition (2.3) (2.4), we find that,

$$
E_{i}=F_{0 i}^{1}+{ }^{*} F_{0 i}^{2}
$$

and

$$
B_{i}={ }^{*} F_{0 i}^{1}-F_{0 i}^{2} .
$$

So it is convenient to define a new field tensor as

$$
\begin{aligned}
& F_{\mu \nu}=F_{\mu \nu}^{1}+{ }^{*} F_{\mu \nu}^{2}, \\
& \widetilde{F}_{\mu \nu}={ }^{*} F_{\mu \nu}^{1}-F_{\mu \nu}^{2},
\end{aligned}
$$

where $\widetilde{F}_{\mu \nu}$ is exactly the Hodge star dual of $F_{\mu \nu}$. As we shall see, using these new field tensors we can easily express the duality symmetry in a compact fashion. It is easy to see that $F_{\mu \nu}$ is the analog to the usual electromagnetic tensor defined in classical electrodynamics, because they have exactly the same matrix form in terms of the field strengths. Since the vector potentials in our formalism have no singularities one has $\partial^{\mu *} F_{\mu \nu}^{I}=0$, so Maxwell's equations can also be written as

$$
\begin{aligned}
& \partial^{\mu} F_{\mu \nu}=\partial^{\mu} F_{\mu \nu}^{1}=J_{\nu}^{1} \\
& \partial^{\mu} \widetilde{F}_{\mu \nu}=-\partial^{\mu} F_{\mu \nu}^{2}=J_{\nu}^{2}
\end{aligned}
$$

One can find that (refer to section 4), from $F_{\mu \nu}$ or $\widetilde{F}_{\mu \nu}$ defined above, we can easily build a Lagrangian such that the Maxwell's equation (2.15) can be derived from the action principle. 


\section{$3 \quad S O(2)$ duality symmetry}

The $S O(2)$ duality symmetry of Electromagnetic field theory has been discussed in many papers [6] [8] [7] [10]. In our previous paper [14], we explained in detail why the general duality symmetry is the $S O(2)$ symmetry, but there still exists something not very clear. For example, under the general dual transformation for $F_{\mu \nu}, \widetilde{F}_{\mu \nu}$, i.e.

$$
\left(\begin{array}{l}
F_{\mu \nu}^{\prime} \\
\widetilde{F}_{\mu \nu}^{\prime}
\end{array}\right)=\left(\begin{array}{ll}
a & b \\
c & d
\end{array}\right)\left(\begin{array}{l}
F_{\mu \nu} \\
\widetilde{F}_{\mu \nu}
\end{array}\right)
$$

why should the same transformation hold simultaneously for $J^{\mu 1}, J^{\mu 2}$ ? One can make the same question concerning the dual transformations of $(\mathbf{E}, \mathbf{B})$ and $(q, g),\left(\mathbf{J}_{e}, \mathbf{J}_{m}\right)$, etc. Why all these dual transformations must be the same? In our formulation, we can shed light on this issue. So in this section we would first like to give answers to these questions and then explain in detail why our formulation has manifestly $S O(2)$ duality symmetry, i.e. we will see that the index $I$ of potentials $A_{\mu}^{I}$ is the $S O(2)$ index.

Let us first solve the Maxwell's equation in our formalism. It is easy to check that the potential functions defined in the section above satisfy the differential equations [14]:

$$
\begin{aligned}
& \frac{\partial^{2}}{\partial t^{2}} \phi_{1}-\nabla^{2} \phi_{1}=\rho_{e}, \\
& \frac{\partial^{2}}{\partial t^{2}} \mathbf{A}_{\mathbf{1}}-\nabla^{2} \mathbf{A}_{\mathbf{1}}=\mathbf{j}_{\mathbf{e}} \\
& \frac{\partial^{2}}{\partial t^{2}} \phi_{2}-\nabla^{2} \phi_{2}=-\rho_{m}, \\
& \frac{\partial^{2}}{\partial t^{2}} \mathbf{A}_{\mathbf{2}}-\nabla^{2} \mathbf{A}_{\mathbf{2}}=-\mathbf{j}_{\mathbf{m}}
\end{aligned}
$$

In the static case, i.e. when the sources do not depend on time $t$, we can write

$$
\rho_{I}=\rho_{I}(\mathbf{x}), \quad \mathbf{J}_{I}=\mathbf{J}_{I}(\mathbf{x}), \quad I=1 \operatorname{and} 2,
$$

where $I=1,2$ represent $I=e, m$ respectively. Then exactly as it is done in the standard classical electrodynamics (magnetic source free case) [11], the solution of equation (3.2) is given by

$$
\begin{gathered}
\phi_{I}=\frac{1}{4 \pi} g^{I I^{\prime}} \int \frac{\rho_{I^{\prime}}\left(\mathbf{x}^{\prime}\right)}{r} d^{3} x^{\prime} \\
\mathbf{A}_{I}(\mathbf{x})=\frac{1}{4 \pi} g^{I I^{\prime}} \int \frac{\mathbf{J}_{I^{\prime}}\left(\mathbf{x}^{\prime}\right)}{r} d^{3} x^{\prime}
\end{gathered}
$$

where $r=\left|\mathbf{x}-\mathbf{x}^{\prime}\right|$, then from equations (2.3) and (2.4) we find that the field strengths have the following representation 


$$
\mathbf{E}(\mathbf{x})=\frac{1}{4 \pi} \int \rho_{e}\left(\mathbf{x}^{\prime}\right) \frac{\mathbf{r}}{r^{3}} d^{3} x^{\prime}+\frac{1}{4 \pi} \int \mathbf{J}_{m}\left(\mathbf{x}^{\prime}\right) \times \frac{\mathbf{r}}{r^{3}} d^{3} x
$$

and

$$
\mathbf{B}(\mathbf{x})=\frac{1}{4 \pi} \int \rho_{m}\left(\mathbf{x}^{\prime}\right) \frac{\mathbf{r}}{r^{3}} d^{3} x^{\prime}-\frac{1}{4 \pi} \int \mathbf{J}_{e}\left(\mathbf{x}^{\prime}\right) \times \frac{\mathbf{r}}{r^{3}} d^{3} x .
$$

Now we can give the answer to the question in the beginning of this section. Because $E_{i}=F_{0 i}, B_{i}=\widetilde{F}_{0 i}$, then we know that if $F_{\mu \nu}, \widetilde{F}_{\mu \nu}$ have a transformation given by equation (3.1), which led to the field strengths $\mathbf{E}, \mathbf{B}$ having the same transformation, and because the field strengths are related to the sources by equation (3.6) and (3.7), so the sources $\rho_{e}, \rho_{m}$ and $\mathbf{J}_{e}, \mathbf{J}_{m}$ must change in the same way. The same transformation must be satisfied by the 4-dimensional currents $J^{\mu 1}, J^{\mu 2}$. That is why once one chooses the transformation for $F_{\mu \nu}, \widetilde{F}_{\mu \nu}$ given by (3.1), then the corresponding field strengths and the the sources must obey the same transformation. If we impose that the Maxwell's equations (2.6) and (2.7) are invariant under these transformations of field strengths and the sources, we obtain $a=d$ and $b=-c$. Moreover, if we also impose that the energy density $\frac{1}{2}\left(\mathbf{E}^{2}+\mathbf{B}^{2}\right)$ and the Poynting vector $\mathbf{E} \times \mathbf{B}$ are invariant under this transformation we get $a^{2}+b^{2}=1$. It is then natural to introduce an angle $\alpha$ such that $a=\cos \alpha$ and $b=\sin \alpha$. Hence the general duality transformation matrix coincides with the general rotation matrix in two dimensions. Thus it becomes apparent that the general electromagnetic duality symmetry is the $\mathrm{SO}(2)$ symmetry. Under the special case, $\alpha=\pi / 2$, the transformation (3.1) coincides with the replacement $F_{\mu \nu} \rightarrow \widetilde{F}_{\mu \nu}, \quad \widetilde{F}_{\mu \nu} \rightarrow-F_{\mu \nu}$ and the same replacements must be taken simultaneously, i.e. $\mathbf{E} \rightarrow \mathbf{B}, \mathbf{B} \rightarrow-\mathbf{E}, \rho_{e} \rightarrow \rho_{m}, \rho_{m} \rightarrow$ $-\rho_{e}$ and $\mathbf{J}_{e} \rightarrow \mathbf{J}_{m}, \mathbf{J}_{m} \rightarrow-\mathbf{J}_{e}$, etc. This corresponds to the usual special electro-magnetic duality symmetry.

Now we would like to point out that the index $I$ of the potentials $A_{\mu}^{I}$ is the $S O(2)$ index. Under the general dual transformation, i.e. $S O(2)$ transformation, from the discussion above we know that the sources $\rho_{I}$ and $\mathbf{J}_{I}$ change as

$$
\begin{gathered}
\rho_{I^{\prime}}=R(\alpha)_{I I^{\prime}} \rho^{I^{\prime}} \\
\mathbf{J}^{\prime}{ }_{I}=R(\alpha)_{I I^{\prime}} \mathbf{J}^{I^{\prime}},
\end{gathered}
$$

where $R(\alpha)$ is the $S O(2)$ rotation matrix. Then from equations (3.4) and (3.5), we know that the potentials $A_{\mu}^{I}=\left(\phi_{I},-\mathbf{A}_{I}\right)$ should have the same $S O(2)$ transformation, that is to say that the index $I$ of potential $A_{\mu}^{I}$ is the $S O(2)$ index. So our formulation in section (2) has manifestly $S O(2)$ duality symmetry. 


\section{Lagrangian formulation of the field}

In this section we will give a lagrangian for the electromagnetic field which gives the right Maxwell's equation in the presence of magnetic source. We will also see that from this lagrangian one can deduce the right Lorentz force formula (refer to the next section).

The Lagrangian of the field is given by

$$
L=-\frac{1}{4}\left(F_{\mu \nu}\right)^{2}-\left(A_{\mu}^{1}+* A_{\mu}^{2}\right) J^{\mu 1}+\left(* A_{\mu}^{1}-A_{\mu}^{2}\right) J^{\mu 2}
$$

where the $* A_{\mu}^{I}$ is defined through

$$
\partial_{\mu} * A_{\nu}^{I}=\frac{1}{2} \epsilon_{\mu \nu}^{\alpha \beta} \partial_{\alpha} A_{\beta}^{I}
$$

From a simple calculation we find

$$
\begin{gathered}
\frac{\partial F^{2}}{\partial\left(\partial_{\mu} A_{\nu}^{1}\right)}=\frac{\partial\left(F_{\alpha \beta} F^{\alpha \beta}\right)}{\partial\left(\partial_{\mu} A_{\nu}^{1}\right)}=4 F^{\mu \nu}, \\
\frac{\partial F^{2}}{\partial\left(\partial_{\mu} A_{\nu}^{2}\right)}=\frac{\partial\left(F_{\alpha \beta} F^{\alpha \beta}\right)}{\partial\left(\partial_{\mu} A_{\nu}^{2}\right)}=4 \widetilde{F}^{\mu \nu} .
\end{gathered}
$$

Notice that $* A_{\mu}^{I}$ is related to the derivative of $A_{\alpha}^{I}$, and also take into account the conservation conditions of the currents (2.10). Then the Euler-Lagrange equation of the Lagrangian defined in (4.1) gives the Maxwell's equation (2.15).

\section{Action principle of the field}

In this section, we would like to give a consistent action principle formulation for the classical electro-magnetic field theory in the presence of the magnetic sources. We can consider a system of dyons which interact with the electro-magnetic field. From the action of the system we expect to get both field equation (2.15) and also the equations of motion of the dyons.

For simplicity, we consider here one dyon with electric charge $q$ and magnetic charge $g$, which moves in the electro-magnetic field (the extension to the many dyons system can be easily done). The action of this system consists of three parts, i.e,

$$
S=S_{p}+S_{I}+S_{F}
$$


where,

$$
S_{p}=-m \int_{\lambda_{1}}^{\lambda_{2}} \sqrt{-g_{\mu \nu} \frac{d x^{\mu}}{d \lambda} \frac{d x^{\nu}}{d \lambda}} d \lambda
$$

is the free action of the dyon, and

$$
\begin{array}{r}
S_{I}=q \int_{\lambda_{1}}^{\lambda_{2}}\left(A_{\mu}^{1}+* A_{\mu}^{2}\right) \frac{d x^{\mu}}{d \lambda} d \lambda-g \int_{\lambda_{1}}^{\lambda_{2}}\left(* A_{\mu}^{1}-A_{\mu}^{2}\right) \frac{d x^{\mu}}{d \lambda} d \lambda \\
=\int_{\Omega}\left(A_{\mu}^{1}+* A_{\mu}^{2}\right) J^{\mu 1} d^{4} x-\int_{\Omega}\left(* A_{\mu}^{1}-A_{\mu}^{2}\right) J^{\mu 2} d^{4} x
\end{array}
$$

is the term of interaction between the dyon and the electro-magnetic field around it. The $J^{\mu I}$ in the above equation are the currents for one particle dyon which have the form

$$
\begin{aligned}
& J^{\mu 1}=q \int \frac{d x^{\mu}}{d \tau} \delta^{4}(x-x(\tau)) d \tau \\
& J^{\mu 2}=g \int \frac{d x^{\mu}}{d \tau} \delta^{4}(x-x(\tau)) d \tau .
\end{aligned}
$$

The last term of the action

$$
S_{F}=-\frac{1}{4} \int_{\Omega} F_{\mu \nu} F^{\mu \nu} d^{4} x
$$

is nothing but the action of the electro-magnetic field.

Let us now vary the potentials as

$$
A_{\mu}^{I} \Rightarrow A_{\mu}^{I}+\epsilon_{I} B_{\mu}^{I}(x),\left.\quad B_{\mu}^{I}\right|_{\Omega}=0
$$

We can check that

$$
\begin{aligned}
& \left.\frac{\partial S_{f}}{\partial \epsilon_{1}}\right|_{\epsilon_{1}=\epsilon_{2}=0}=\int_{\Omega} \partial_{\mu} F^{\mu \nu} B_{\nu}^{1} d^{4} x \\
& \left.\frac{\partial S_{f}}{\partial \epsilon_{2}}\right|_{\epsilon_{1}=\epsilon_{2}=0}=\int_{\Omega} \partial_{\mu}^{*} F^{\mu \nu} B_{\nu}^{2} d^{4} x
\end{aligned}
$$

Noticing that $\frac{\partial * A_{\mu}^{I}}{\partial \epsilon^{I^{\prime}}}=0$ and $\frac{\partial S_{p}}{\partial \epsilon_{I}}=0$, we then have

$$
\begin{aligned}
& 0=\left.\frac{\partial S}{\partial \epsilon_{1}}\right|_{\epsilon_{1}=\epsilon_{2}=0}=\int_{\Omega}\left(\partial_{\mu} F^{\mu \nu}-J^{\nu 1}\right) B_{\mu}^{1} d^{4} x, \\
& 0=\left.\frac{\partial S}{\partial \epsilon_{2}}\right|_{\epsilon_{1}=\epsilon_{2}=0}=\int_{\Omega}\left(\partial_{\mu} \widetilde{F}^{\mu \nu}-J^{\nu 2}\right) B_{\mu}^{1} d^{4} x .
\end{aligned}
$$

Because $B_{\mu}^{1}$ and $B_{\mu}^{2}$ are arbitrary, then from the equations (5.10) and (5.11) above we reobtain the Maxwell's equation (2.15).

Further if we change the coordinate of the dyon in the form

$$
x^{\mu} \Rightarrow x^{\mu}+\epsilon_{0} y^{\mu}, \text { with } y^{\mu}\left(\lambda_{1}\right)=y^{\mu}\left(\lambda_{2}\right)=0,
$$


we find

$$
\left.\frac{\partial S}{\partial \epsilon_{0}}\right|_{\epsilon_{0}=0}=\int_{\tau_{1}}^{\tau_{2}}\left(-m \frac{d^{2} x^{\alpha}}{d \tau^{2}}+q F_{\beta}^{\alpha} \frac{d x^{\beta}}{d \tau}-g \widetilde{F}_{\beta}^{\alpha} \frac{d x^{\beta}}{d \tau}\right) y_{\alpha} \cdot d \tau
$$

Since $y_{\alpha}$ is arbitrary, then from $\left.\frac{\partial S}{\partial \epsilon_{0}}\right|_{\epsilon_{0}=0}=0$ we obtain

$$
m \frac{d^{2} x^{\alpha}}{d \tau^{2}}=q F_{\beta}^{\alpha} \frac{d x^{\beta}}{d \tau}-g \widetilde{F}_{\beta}^{\alpha} \frac{d x^{\beta}}{d \tau} .
$$

This is just the equation of motion of a dyon moving in the electro-magnetic field. From this equation we can find that the Lorentz force the dyon acquired in the magnetic field can be represented in terms of field strengths as

$$
\mathbf{F}=q(\mathbf{E}+\mathbf{v} \times \mathbf{B})+g(\mathbf{B}-\mathbf{v} \times \mathbf{E}) .
$$

We would like to stress that the general Lorentz force has also the $S O(2)$ electromagnetic duality symmetry.

\section{Conclusion remarks}

The main results of this paper are as follows. First we use the formulation of reference [14 to explain why the classical electro-magnetic field theory in the presence of a magnetic source has exactly the $S O(2)$ duality symmetry. Then we find a proper Lagrangian formulation for the theory, and at last using the action principle of the system of dyons we derived both Maxwell's equation and the equation of motion for the dyon. From this equation of motion we got the general Lorentz force for a dyon moving in the electromagnetic field.

As a consistency check of our formulation we see that for $g=0$ and $J^{\mu 2}=0$ (no magnetic sources), from equation (3.2) we can set $A_{\mu}^{2}=0$, and so $F_{\mu \nu}=F_{\mu \nu}^{1}+{ }^{*} F_{\mu \nu}^{2} \Rightarrow F_{\mu \nu}^{1}$. This means that our formulation contains standard electrodynamics as a particular case. For $q=0$ and $J^{\mu 1}=0$ (no electric sources), one has $A_{\mu}^{1}=0$, and then $F_{\mu \nu}=F_{\mu \nu}^{1}+{ }^{*} F_{\mu \nu}^{2} \Rightarrow^{*}$ $F_{\mu \nu}^{2}$, and the lagrangian becomes:

$$
L=-\frac{1}{4}\left({ }^{*} F_{\mu \nu}^{2}\right)^{2}-A_{\mu}^{2} J^{\mu 2}=\frac{1}{4}\left(F_{\mu \nu}^{2}\right)^{2}-A_{\mu}^{2} J^{\mu 2} .
$$

Thus in this case the formulation is completely parallel to the magnetic source free case. 
Acknowledgements. We would like to thank S. Carneiro for his kind and valuable comments and for letting us know reference [5]. Part of this work is done during K. Li 's research visiting to Abdus Salam International Centre for Theoretical Physics, Trieste Italy. This work was partially supported by the National Nature Science Foundation of China and the Consejo Nacional de Investigaciones Científicas y Técnicas (CONICET), Argentina.

\section{References}

[1] Elias Kiritsis, Supersymmetry and Duality in field theory and string theory, hepth/9911525, and the references there in.

[2] J. H. Schwarz and N. Seiberg, Rev. Mod. Phys.71 (1999) S112.

[3] R.A.Brandt, F.Neri and D.Zwanziger, Phys. Rev. D 19 (4) (1979) 1153.

[4] P. A. M. Dirac, Proc. R. Soc. London A133 (1931) 60.

[5] Cardoso de Mello, S. Carneiro and M.C.Nemes, Phys. Lett. B384(1996)197; S. Carneiro, J. High Energy Physics 07(1998)022

[6] K. Lechner and P.A. Marchetti, hep-th/9906079.

K. Lechner, "A quantum field theory of dyons", hep-th/0003003.

[7] L. Gamberg and K. A. Milton, Phys. Rev. D 61 (2000) 75013.

[8] J. Méndez, "Electromagnetic Duality based on Axiomatic Maxwell Equations", hepth/9911188.

[9] C. A. P. Galvao and J. A. Mignaco, "A consistent electromagnetic duality", hepth/0002182.

[10] S. Donev "A new look on EM Duality. Suggestions and developments", hepth/0006208.

[11] J. D. Jackson, "Classical Electrodynamics", Ed. John Wiley, Singapore, 1999.

[12] B. Felsager, "Geometry, Particles and Fields", Springer-Verlag, New York, 1998.

[13] C. Itzykson and J. B. Zuber, "Quantum Field Theory", Ed. Mc. Graw-Hill Inc., 1980. 
[14] Kang Li and Carlos M. Naón, "An alternative formulation of classical electromagnetic duality", Mod. Phys. Lett. A 16 (2001) 1671. 